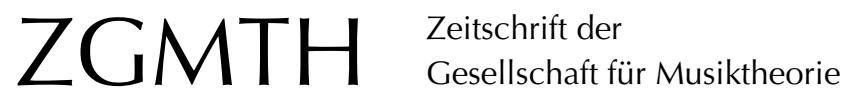

Sprick, Jan Philipp (2013): Wiederholung und Symmetrie im Kopfsatz von Johannes Brahms' Sonate Es-Dur op. 120/2. ZGMTH 10/1, 147-158.

https://doi.org/10.31751/718

(C) 2013 Jan Philipp Sprick

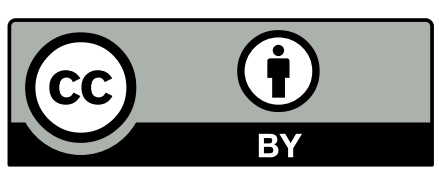

Dieser Text erscheint im Open Access und ist lizenziert unter einer Creative Commons Namensnennung 4.0 International Lizenz.

This is an open access article licensed under a

Creative Commons Attribution 4.0 International License.

veröffentlicht / first published: 06/07/2014

zuletzt geändert / last updated: 19/02/2016 


\title{
Wiederholung und Symmetrie im Kopfsatz von Johannes Brahms' Sonate Es-Dur op. 120/2
}

\author{
Jan Philipp Sprick
}

\begin{abstract}
Entgegen einer Tendenz in der Brahms-Forschung, die Aspekte `Entwicklung und 'Variation in den Mittelpunkt zu rücken, fragt der vorliegende Beitrag nach der Rolle von ,Wiederholung und 'Symmetries in Brahms später Sonate op. 120/2 für Klarinette (oder Bratsche) und Klavier. Auf einer kleinformalen, satztechnischen Ebene zeigt sich Wiederholung in erster Linie in wiederkehrenden Satzmodellen und in sequenziellen Strukturen. Auch hinsichtlich der großformalen Gestaltung ist der Kopfsatz der Sonate stark von Wiederholungen geprägt, die sich jedoch weniger an der Oberfläche, als vielmehr subkutan manifestieren.
\end{abstract}

Die Rezeption von Arnold Schönbergs Aufsatz »Brahms der Fortschrittliche«" leitete einen Paradigmenwechsel in der analytischen Auseinandersetzung mit dem Werk von Johannes Brahms ein. Seitdem ist die Behauptung, Schönbergs Begriff der sentwickelnden Variation biete eine angemessene Beschreibung Brahms' motivisch-thematischer Verfahrensweisen, zu einem zentralen Topos der Brahms-Analytik geworden. ${ }^{2}$ Der Übergang zur Neuen Musik wird vor diesem Hintergrund nicht als Bruch mit der Vergangenheit, sondern als Konsequenz bestimmter kompositorischer Tendenzen des 19. Jahrhunderts interpretiert. Allerdings hat die emphatische Behauptung einer direkten Traditionslinie von Brahms zu Schönberg immer wieder auch berechtigte Kritik erfahren. ${ }^{3}$ Zwar ist nicht zu leugnen, dass Brahms' dichte motivische Prozesse, asymmetrische Phrasenstrukturen und komplexe Harmonik vieles von dem antizipieren, was auch Schönberg kompositorisch anstrebte, doch erscheint die mit dem Schönberg'schen `Wiederholungsverbot $\iota$ zusammenhängende affirmative Bewertung von `Entwicklung als zu einseitig.

Schönberg selbst kritisiert in seinen Schriften nicht das Prinzip der Wiederholung an sich, sondern »unveränderte« oder »leicht variierte Wiederholungen, die sich in nichts Wesentlichem vom ersten Auftreten unterscheiden«. So beobachtet er bei Brahms, dass »Phrasen, Motive und andere strukturelle Bestandteile von Themen nur in variierter Form« wiederholt werden, vorzugsweise »in der Form dessen, was ich entwickelnde Variation nenne ${ }^{4}{ }^{4}$

1 Schönberg 1976a.

2 Vgl. Schmidt 1971 und Frisch 1984.

3 Vgl. etwa Schmidt, der in einer solchen Position etwa das »Konstrukt einer akademischen Geschichtsbetrachtung« erkennt (2000, 8). Vgl. auch Reiter 2000, 15.

4 Schönberg 1976b, 126 (Hervorhebung original). 
Vor diesem Hintergrund sollen am Beispiel des Kopfsatzes der Sonate op. 120/2 zwei Aspekte untersucht werden, die von der Brahms-Forschung bislang nur am Rande thematisiert worden sind: Wiederholung und Symmetrie. ${ }^{5}$ Dabei wird die Wiederholung als formbildendes Prinzip sowohl auf makro- als auch auf mikroformaler Ebene in den Blick genommen. Letztere wird insbesondere in sequenziellen Strukturen wirksam. Dass Sequenzen in Brahms' Musik bislang nur geringe Aufmerksamkeit zuteil geworden ist, dürfte eine Ursache auch in Schönbergs vielzitierter Bezeichnung der Sequenz als »minderwertige Konstruktionsmethode « haben. ${ }^{6}$ Gerade die Verbindung harmonischer Sequenzbildungen mit variativen Verfahren ist jedoch ein wesentlicher Aspekt Brahms'scher Poetik. Auch in Bezug auf die Form erscheint eine einseitig auf ,Entwicklung، ziehende Betrachtung als zu kurz gegriffen. So bezeichnet Schönberg es als Aufgabe der Form, »Faßlichkeit durch Erinnerbarkeit zu bewirken«. ${ }^{7}$ Hinsichtlich der Mittel, mit Hilfe derer 'Fasslichkeitı erreicht werden soll, stellt er jedoch weniger die Entwicklung musikalischer Gedanken ins Zentrum, als vielmehr die Prinzipien »Ausgewogenheit, Regelmäßigkeit, Symmetrie, Unterteilung, Wiederholung, Einheit rhythmische und harmonische Beziehungen und sogar Logik«. ${ }^{8}$

\section{Gesamtanlage}

In Bezug auf die Gesamtanlage des Satzes ist das symmetrische Längenverhältnis der drei Hauptteile auffällig. ${ }^{9}$ In der Literatur werden im Hinblick auf die zwei den Formverlauf entscheidenden Fragen nach dem Beginn des Seitensatzes und dem Beginn der Durchführung sehr unterschiedliche Auffassungen vertreten, ohne dass die Differenzen argumentativ zueinander in Beziehung gesetzt würden. So wird der Beginn des Seitensatzes mal in Takt 22 (Webster 1990 und Krämer 2009) und mal in Takt 40 (Reiter 2000 und Edelmann 2013) verortet. Ähnlich verhält es sich mit dem Durchführungsbeginn, der wahlweise in Takt 52 (Reiter 2000 und Edelmann 2013) oder in Takt 56 (Adrian 1990, Webster 1990 und Krämer 2009) angesetzt wird. Während beispielsweise Reiter davon ausgeht, dass die »nicht ausgeprägte Zäsur« zwischen Schlussgruppe und Durchführung den »Charakter der Durchführung als Prolongation der etablierten Dominantspannung « erhöhe, erkennt Adrian im Kopfsatz von op. 120/2 eine »ternary sonata-form«. Diese zeichne sich dadurch aus, dass der Durchführungsbeginn, der aus Adrians Sicht in Takt 56 anzusetzen ist, einen erneuten Anlauf von der Tonika nimmt. Dies stelle das ssonata

5 Man muss nicht so weit gehen wie Christian Martin Schmidt und die beiden Sonaten op. 120 in ihrer Eigenschaft als letzte Kammermusikwerke des Komponisten als »bündelnden Rückblick auf dessen gesamtes Komponieren« bezeichnen (1994, 157), um in den Stücken exemplarische Eigenschaften von Brahms' Komponieren zu erkennen. Gerade die Sonate op. 120/1 ist vielfach für die Darstellung grundsätzlicher Aspekte der Brahms'schen Poetik herangezogen worden: vgl. Schmidt 1971, Frisch 1984, Reiter 2000 und Boestfleisch 2001.

6 Schönberg 1976b, 128. Vgl. dazu allgemein auch Sprick 2012. Eine Ausnahme ist ein jüngerer Beitrag von Ryan McClelland (2012) zur Sequenz als »expressive culmination« bei Brahms.

7 Schönberg 1976a, 36.

8 Ebd.

9 Exposition: 51 Takte; Durchführung: 52 Takte; Reprise: 47 Takte; Coda: 24 Takte. 
principle`, die übergeordnete Zweiteiligkeit des Sonatensatzes, in Frage ${ }^{10}$, da die (etwa von Reiter postulierte) Prolongation der Dominantspannung in die Durchführung hinein nicht aufrechterhalten werde. ${ }^{11}$ Dementsprechend wird auch der Beginn der Coda, der strukturell mit dem Durchführungsbeginn zusammenhängt, entweder in Takt 150 (Reiter 2000 und Edelmann 2013) oder in Takt 154 (Adrian 1990, Webster 1990 und Krämer 2009) angesetzt. Als möglichen Ausgangspunkt für eine Klärung dieser offensichtlichen Widersprüche diskutiere ich im Folgenden die Bedeutung des dem Hauptsatz zugrunde liegenden Oktavregelmodells für den Formverlauf.

\section{Materialebenen}

Der Formverlauf des ersten Satzes ist subkutan von zwei Materialebenen geprägt, die beide in der Exposition angelegt sind und zunächst unabhängig voneinander verlaufen. Die wichtigere dieser beiden Ebenen ist das Oktavregelmodell des Hauptsatzes, das im Satzverlauf regelmäßig wiederkehrt (Beispiel 1). Vergleicht man die verschiedenen Realisierungen des Modells in Reprise und Coda mit denjenigen in Exposition und Durchführung, so legen die auf Wiederholung basierenden strukturellen Entsprechungen eine zweiteilige Gliederung des Satzes in Exposition und Durchführung einerseits sowie in Reprise und Coda andererseits nahe (Beispiel 1).

Eine zweite Materialebene des Satzes, die sich in gewisser Weise gegenläufig zur Regelmäßigkeit der Oktavregelharmonik verhält, ist eine Folge von charakteristischen harmonischen Wendungen. Deren Abfolge prägt auf den gesamten Satz gesehen keine Wiederholungsstruktur wie die Oktavregelausschnitte, sondern vielmehr eine Bogenform aus (Beispiel 2).

Die zwei Trugschlüsse in den Takten 92f. und 153f. werden von übermäßigen Quintsextakkorden gerahmt. ${ }^{12}$ Auffällig ist hier, dass der in Takt $21 \mathrm{f}$. ausgesparte - für die reguläre Weiterführung des Klangs aber typische - Quartsextakkord in Takt 161 erscheint. Der dort fehlende Quintfall wiederum ist in Takt 22 vorhanden, so dass sich beide Progressionen wechselseitig ergänzen. Im Gegensatz zu der subkutanen Strukturierung des Satzverlaufs durch das Oktavregelmodell erfüllen diese scharakteristischen Wendungen ihre strukturierende Funktion an der Oberfläche. Beide Materialebenen laufen zunächst unabhängig voneinander und werden erst in der Coda verbunden, wo die trugschlüssige Wendung in den Takten 153/54 die beiden letzten Oktavregelmodelle miteinander verklammert.

10 Vgl. Cone 1968. Auch aus schenkerianischer Perspektive ist die Sonatenform prinzipiell zweiteilig.

11 Adrian 1990, 58. Adrian nennt weitere Beispiele für »ternary sonata-forms « bei Brahms: Violinsonate Nr. 1 G-Dur, op. 78; Sinfonie Nr. 4 e-Moll, 1. Satz; Klaviertrio C-Dur, op. 87, 1. Satz.

12 Es gibt eine weitere trugschlüssige Wendung in Takt 18, die aber eine weniger starke formale Gliederungsfunktion hat und daher in dieser Übersicht nicht berücksichtigt wird. 


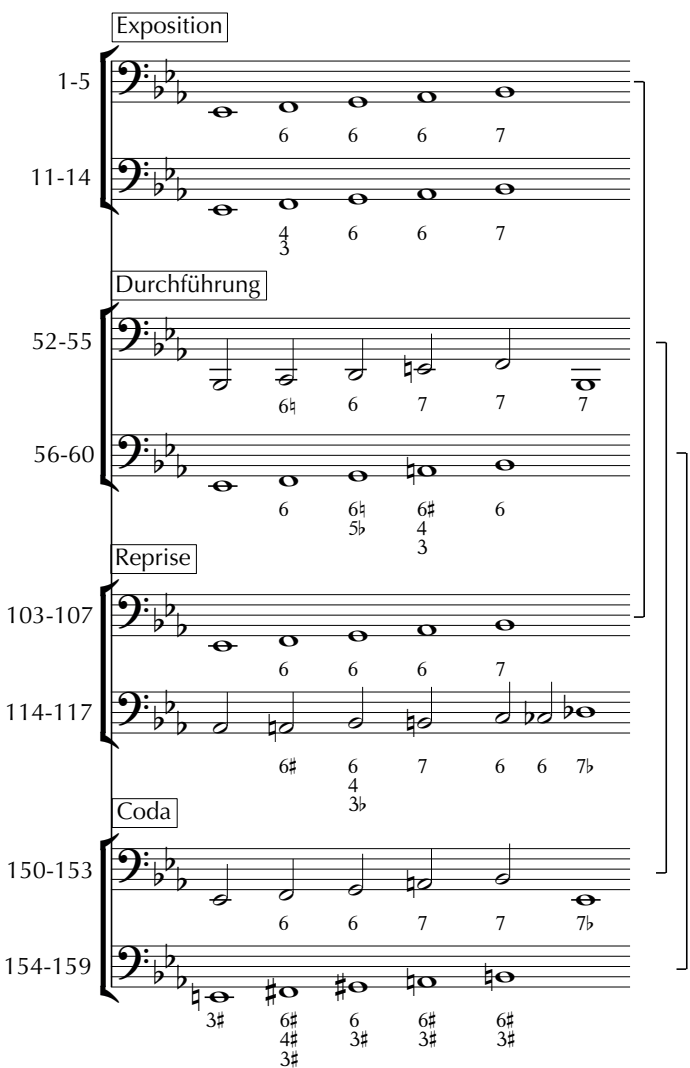

diatonischer Oktavregelausschnitt I-V

diatonischer Oktavregelausschnitt I-V

Oktavregel mit erhöhter IV. Stufe und Quintfall

V. Stufe als Sextakkord

diatonischer Oktavregelausschnitt I-V

»Dynamisierung« mit Zwischendominanten

Oktavregel mit erhöhter IV. Stufe und Quintfall

V. Stufe als Sextakkord

Beispiel 1: Johannes Brahms, Sonate Es-Dur op. 120/2, 1. Satz, Modifikationen des Oktavregelmodells

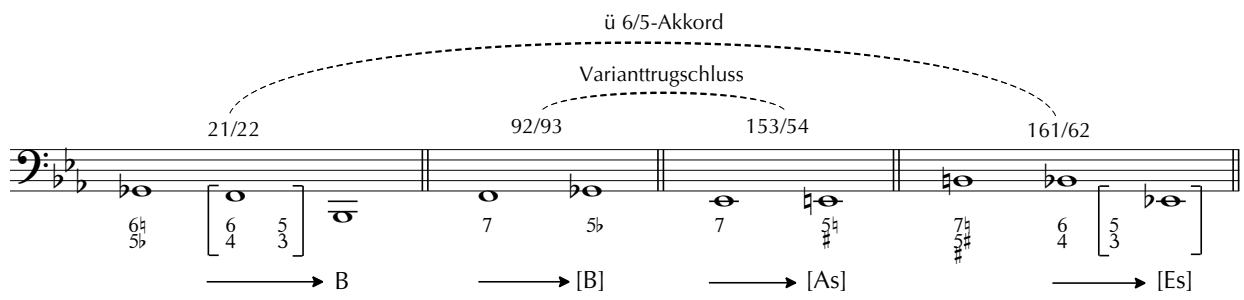

Beispiel 2: Johannes Brahms, Sonate Es-Dur op. 120/2, 1. Satz, harmonische Wendungen als Ausgangspunkte für formbildende Rückschlüsse

\section{Exposition}

Das zehntaktige, liedhafte Thema hebt ohne Vorspiel des Klaviers in der Klarinette an. Über dem Oktavregelmodell entfaltet sich die Kantilene der Klarinette mit einer sukzessi- 
ven Ambituserweiterung bis zum Höhepunkt in Takt 5. Die zunächst achttaktige Periode endet halbschlüssig in Takt 8, gefolgt von einem zweitaktigen Appendix, der sich durch das aufwärts gerichtete Klarinetten-Arpeggio öffnet. Es handelt sich hier also weniger um eine Vordersatz-Nachsatz-Konstruktion als vielmehr um die Fortspinnung eines Vordersatzes (T. 1-4) durch eine sequenzielle Entwicklungsphrase ab Takt 5 sowie einen kadenziellen Appendix. In den Takten 11-15 kehrt der harmonische Verlauf der Takte 1-5 wieder. Mit diesem doppelten Auftreten des Oktavregelmodells wird eine Konstellation etabliert, die - wie oben beschrieben - den weiteren Verlauf des Satzes prägt.

Das kanonische Überleitungsthema wird in Takt 22, unter Auslassung der Dominante F-Dur, durch den übermäßigen Quintsextakkord über ges erreicht. Dieser zwischen Haupt- und Seitenthema auftretende Kontrastgedanke, dessen Material weite Teile der Durchführung beherrscht, hat fraglos Themencharakter. ${ }^{13}$ Zwar ruft dieser Abschnitt, wie auch der spätere Seitensatz, die V. Stufe auf, allerdings wird B-Dur hier, anders als im Seitensatz, nicht über eine perfekte Kadenz erreicht, und die analoge Passage in der Reprise steht nicht in Es-, sondern in Ces-Dur. Die formale Bedeutung dieses Teils bleibt daher ambivalent und spielt mit den Hörerwartungen innerhalb des Modells der dualistischen Sonatensatzform: Den Charakter des Überleitungsthemas gestaltet Brahms kontrastierend zum Hauptsatz und trennt das Thema durch einen `Ausbruch im Klavier - ähnlich wie später auch den Seitensatz - vom vorherigen Geschehen ab. Ab Takt 30 stagniert der Satz auf einem F-Orgelpunkt, wobei die sukzessive Anreicherung des Begleitrhythmus zur Spannungszunahme beiträgt. Ein erneutes Klaviersolo mündet in eine deutlich artikulierte Kadenz nach B-Dur, die das Geschehen des Überleitungsteils zugleich bündelt und abschließt. Hierauf beginnt in Takt 40 der eigentliche Seitensatz.

Während Hauptsatz und Überleitungsthema in satztechnischer Hinsicht von der Oktavregel und dem Kanon bestimmt waren, ist der Seitensatz sequenziell geprägt und weist damit auf die Sequenz als dominierende Satztechnik der Durchführung voraus. Die thematische Substanz lässt sich aus dem ersten Thema ableiten und als Variation der Eröffnungsphrase verstehen. Durch die Sequenzierung gewinnt das Thema einen stärker fließenden und zielgerichteten Charakter als der Hauptsatz, auch wenn die (an vielen Stellen im Satz auftretende) Verkürzung formaler Einheiten den extrem knapp gefassten Seitensatz als fragmentarische Andeutung erscheinen lässt. Wie in vielen anderen Seitensätzen von Brahms zeigt sich auch hier eine rhythmische Belebung, die gemeinsam mit der sequenziellen Struktur den fragmentarischen Charakter konterkariert, der durch das Abbrechen der melodischen Entwicklung entsteht.

Die Reduktion auf den Gerüstsatz (Beispiel 3) zeigt die Kombination einer 7-6-Kette (T. 44/45) und einer Quintfall-Variante mit Sekundakkorden (T. 46/47). Der Zielklang D-Dur in Takt 48 könnte allerdings, an identischer metrischer Position, auch nach einer regelmäßigen Fortsetzung der 7-6-Kette über einen phrygischen Halbschluss erreicht werden, was die sequenzielle Anlage des Seitensatzes noch zusätzlich unterstreicht. ${ }^{14}$

13 Vgl. seine entsprechende Interpretation als Seitensatz in Webster 1990 und Krämer 2009.

14 Die Zieltonart D-Dur wird zwar ganzschlüssig erreicht, die phrygische Einkadenzierung jedoch auf der zweiten Takthälfte von Takt 48 nachgereicht. 


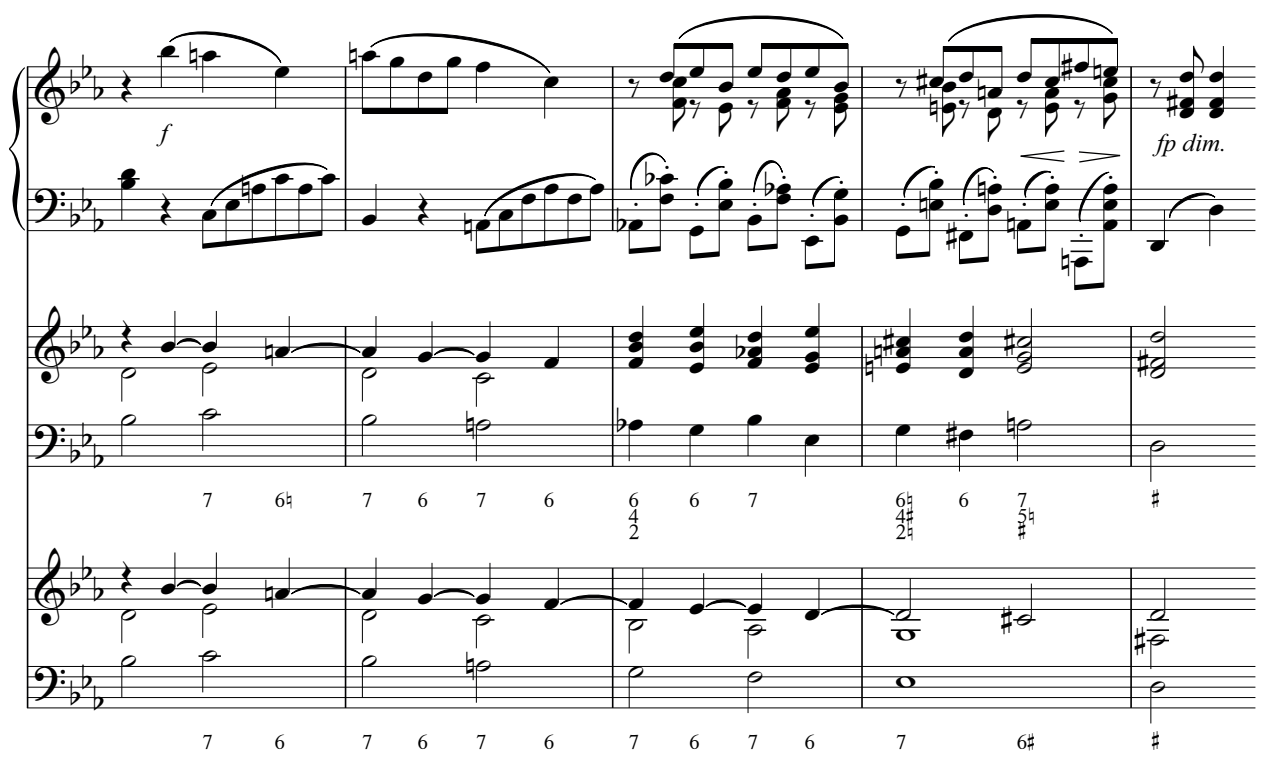

Beispiel 3: Johannes Brahms, Sonate Es-Dur op. 120/2, 1. Satz, Modellkonfiguration im Seitensatz, T. 44-48

Durch motivische Analogien zwischen Haupt- und Seitensatz erscheint die Exposition als durch die zwei kurzen `Ausbrüche` im Klavier unterteilte A-B-A'-Form. ${ }^{15}$ Durch diese formale Gestaltung ist die gesamte Anlage der Exposition von variierter Wiederholung geprägt. Eine kurze Schlussgruppe (T. 48ff.) beschließt die Exposition.

\section{Durchführung}

Die Frage, ob man den Beginn der Durchführung in Takt 52 oder Takt 56 verortet, hängt davon ab, welches Gewicht man einerseits der wiederkehrenden Abfolge der beiden Oktavregelmodelle (T. 52) und andererseits dem in der rechten Hand des Klaviers artikulierten Neuansatz des Hauptthemas als Durchführungsbeginn (T. 56) zuerkennen möchte. So zeigt sich in den Takten 52-55 eine nur schwer aufzulösende Ambivalenz von

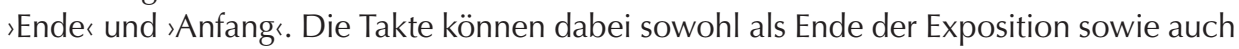
als Beginn der Durchführung gehört werden. Die Wiederholung der Oktavregelmodelle wirkt hier nur subkutan und lässt deren formal gliedernden Aspekt in den Hintergrund treten. Damit überträgt Brahms seine Technik, regelmäßige Sequenzbildungen durch variative Modifikationen an der Oberfläche zu verschleiern, auf die Ebene des Formverlaufs. Exposition und Durchführung sind nicht deutlich voneinander abgegrenzt, sondern gehen in einer Art auskomponierter Überleitung ineinander über. Damit wird in diesem Satz nicht, wie so oft bei Brahms, der Reprisenansatz, sondern der Durchführungsbeginn

15 Vgl. dazu Falke 1997, 177. 
mit dem Ende des vorherigen Formteils verschränkt. ${ }^{16}$ Dieses Verfahren stützt die bereits erwähnte, übergeordnete zweiteilige Gliederung.

Die Beruhigung der Musik - analog zum Vorfeld des Überleitungsthemas in der Exposition - und der Umstand, dass eine Expositionswiederholung, auf die Brahms in diesem Satz ausnahmsweise verzichtet, exakt an Takt 55 anschließen könnte, spräche für einen Durchführungsbeginn in Takt 56. Eine an den Wiederholungen der Oktavregelmodelle orientierte Interpretation des Formverlaufs, wie sie hier vorgeschlagen wird, muss den Beginn der Durchführung hingegen in Takt 52 ansetzen. ${ }^{17}$

Die dreiteilig angelegte Durchführung ist in vielerlei Hinsicht von Wiederholungen geprägt. Auf die variierte Wiederholung von Teilen des Expositionsablaufs (T. 52-72) folgt ein von Sequenzflächen dominierter Abschnitt (T. 73-92), der schließlich in eine harmonisch komplexe Rückleitung mündet (T. 93-102). Beide im ersten Teil der Durchführung auftretenden Oktavregelmodelle (vgl. Beispiel 2) verwenden die erhöhte IV. Stufe. ${ }^{18}$ Nach dem halbtaktig beschleunigten Anstieg zur V. Stufe von B-Dur in den Takten $52 \mathrm{ff}$. erklingt die Melodie des Hauptsatzes über der von es aus steigenden Basslinie in Takt 59. Die fünfte Bassstufe von Es-Dur als Zielebene der Bewegung in Takt 60 wird allerdings nicht als Dominantgrundton aufgefasst (wie in Takt 5), sondern als Terz von g-Moll. Der sich daran anschließende sequenzielle Terzstieg - der von Material und Gestus auf Takt $15 \mathrm{ff}$. rückschließt - führt trugschlüssig nach c-Moll in Takt $63 .{ }^{19} \mathrm{Nach}$ dem Einsatz der Klarinette in Takt 63, der die Takte 18-20 von drei auf zwei Takte komprimiert, wird g-Moll ab Takt 65 durch Material des Überleitungsthemas im Klavier als neues tonales Zentrum etabliert und ab Takt 69 nach G-Dur aufgehellt. Bis hierhin erreicht Brahms also zweierlei: einen wiederholenden Kurzdurchgang durch den Ablauf der Exposition (Hauptsatz und Überleitungsthema) und die sukzessive Etablierung der III. Stufe als neues tonales Zentrum.

Ab Takt 73 orientiert sich die Durchführung nicht mehr am Formverlauf der Exposition, sondern geht in einen flächig-sequenziellen Abschnitt über, der nach einem Pendel zwischen B und Es in Takt 89 den Durchführungshöhepunkt erreicht. Die folgende leitereigene Monte-Sequenz (F-B, G-C, A-d) über einem F-Orgelpunkt baut eine harmonische Spannung auf, die sich in Takt 92/93 jedoch nicht in die erwartete Kadenz zur Reprisenvorfeldebene B auflöst, sondern trugschlüssig nach Ges-Dur gewendet wird. Die erwartete Kadenz nach B-Dur wäre - nach einer Vielzahl von plagalen und halbschlüssigen Wendungen in der ersten Hälfte der Durchführung - die erste ganzschlüssige Wendung in der Durchführung gewesen. Nach den parenthetischen Takten 93-98 wird schließlich das dominantische Orgelpunkt-Reprisenvorfeld in Takt 99 mit Hilfe einer realen Sequen-

16 Beispiele, in denen die harmonische und die motivische Reprise dissoziiert werden, sind das Streichquartett op. 51/1, T. 133ff. und der Reprisenbeginn im ersten Satz der 4. Sinfonie op. 98, T. 246 ff.

17 Adrian macht an dem von ihm postulierten Durchführungsbeginn in Takt 56 die »ternary-sonata-form« fest, die sich aus dem dreimaligen Ansatz von der I. Stufe aus ergibt. Dadurch fehlt in diesen Sätzen der "all-important sense of a continuous sweep from bar 1 to the point of recapitulation «, auch wenn die für die gesamte Satzstruktur wesentliche Unterbrechung am Reprisenbeginn präsent ist. (1990, 78)

18 Krämer $(2009$, 471) betont die wichtige Rolle der erhöhten IV. Stufe für die Etablierung von B-Dur als annähernd gleichwertiger Tonart.

19 Vgl. Takt 18 der Exposition. 
zierung erreicht, die als konsequente Fortsetzung der durchgehenden Sequenzierung der Durchführung den Kulminationspunkt dieses Formteils darstellt.

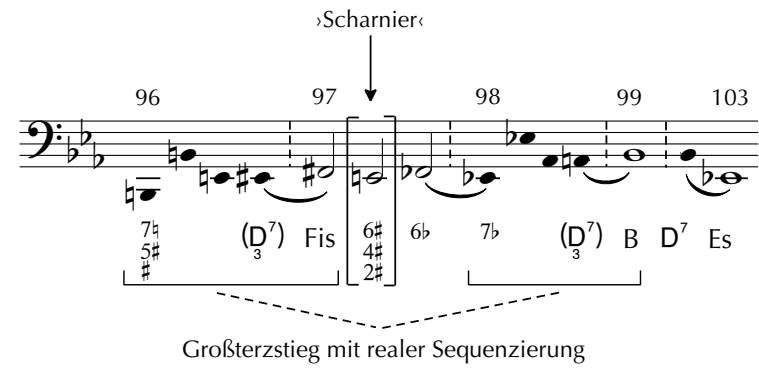

Beispiel 4: Johannes Brahms, Sonate Es-Dur op. 120/2,1. Satz, Ausschnitt Reprisenrückleitung, T. 96-103

Das ıScharnier für diese reale Sequenz ist Takt 97, in dem der auf der ersten Zählzeit erreichte Fis-Dur-Akkord auf der dritten Viertel mit einem fes, der enharmonisch verwechselten Septime e, latent zu einem Sekundakkord wird. Der auf diesen Sekundakkord folgende Fes-Dur-Sextakkord löst sich - und das ist der entscheidende Schritt - in den Es-Dur Septakkord in Takt 98 auf. Es schließt sich die reale Großterz-Sequenzierung von Takt 96 an, so dass analog zu dem Fis-Dur in Takt 97 in Takt 99 das Orgelpunktvorfeld B-Dur erreicht wird. Der Weg von der hochchromatischen Fis-Ebene in die tiefchromatische B-Ebene des Reprisenvorfeldes wird so auf engstem Raum zurückgelegt. ${ }^{20}$ Integriert in diese sequenzielle Fortschreitung ist eine Ambivalenz der Fis-Dur und B-Dur-Akkorde in Takt 97 und Takt 99 zwischen halbschlüssiger und plagaler Wirkung. Diese Ambivalenz von halb- und ganzschlüssiger Wendung ist ein Element, das die Durchführung - wie erwähnt - bereits ab Takt 78 prägt. Vor dem Hintergrund, dass Brahms über einen längeren Zeitraum ganzschlüssige Wendungen meidet, inszeniert er den Reprisenbeginn, den Reiter als »spiegelsymmetrische[s] Korrelat zum Halbschluß E-B am Reprisenhöhepunkt« bezeichnet ${ }^{21}$, umso deutlicher.

Brahms wendet hier also nicht, wie sonst häufig, das Verfahren einer Reprisenverschleierung an. Das Verfahren, den Übergang von zwei Formteilen zu verschleiern, findet sich - wie oben bereits erläutert - stattdessen zu Beginn der Durchführung. Statt einer großen Steigerung bestimmt ab Takt 99 eine dynamische Zurücknahme bis ins ımolto dolce` das Geschehen und bildet damit einen deutlichen Gegensatz zu der bis dahin größten harmonischen Spannung des Satzes in den unmittelbar vorangehenden Takten 93-98.

20 Beide Akkorde auf der zweiten Takthälfte des 'Scharnier`-Taktes 97, der Sekundakkord und der Sextakkord, können als phrygische Auflösungsvarianten in den Dur-Septakkord in Takt 98 fortgeführt werden, der dann als Dominante zu einer imaginären As-Ebene fungiert. Bei Brahms erscheinen Sekund- und Sextakkord hintereinander, bevor die Auflösung in den Es-Dur-Septakkord erfolgt. Die Auflösungsvariante des Sekundakkordes ist jedoch als die übergeordnete Progression an dieser Stelle anzusehen. Für diese phrygische Sekundakkord-Auflösungsvariante gibt es in der musiktheoretischen Literatur des 18. Jahrhunderts viele Beispiele. Auch Carl Philipp Emanuel Bach erwähnt diese Sekundakkordauflösungsvariante in seinem Versuch: In dem Kapitel Vom Secundenaccord schreibt er unter § 6: »Wenn die übermäßige Quarte bey der grossen Secunde und grossen Sexte ist, so kann sie hernach liegen bleiben, und in die Höhe gehen« (Bach 1753/62, 100).

21 Reiter 2000, 191. 


\section{Reprise}

Durch die beschriebenen Elemente beleuchtet Brahms den Reprisenbeginn - den bei Brahms vielleicht entscheidenden Moment jedes Sonatensatzes ${ }^{22}$ - äußerst subtil und gibt dem Thema durch die triolische Begleitung ab Takt 103 einen stärker fließenden Gestus als zu Beginn des Satzes. Der Seitensatz kehrt in den Reprisen der späten Sonatensätze von Brahms stets in ungekürzter und substantiell nicht veränderter Form wieder. ${ }^{23}$ Da die Reprise jedoch fast immer kürzer ist als die Exposition, betreffen die notwendigen Kürzungen meist den Hauptsatz. ${ }^{24}$

Der Eindruck einer >Dynamisierung ‘ des musikalischen Geschehens bei diesem Neuansatz entsteht aber nicht nur durch die triolische Begleitstruktur ab Takt 103, sondern auch durch die Modifikation des zweiten Oktavregelmodells ab Takt 114, das die in der Durchführung bereits aufgetretene Erhöhung der IV. Stufe zu einer zwischendominantischen Chromatik im Bass ausbaut (vgl. Beispiel 1). Der variierte Wiederholungscharakter des Oktavregelmodells in der Exposition und die Nutzung zweier unterschiedlicher Oktavregelmodelle zur Verschleierung des Durchführungsbeginns wird hier im Sinne eines nach vorne gerichteten Spannungsaufbaus modifiziert. Anstelle einer Reihung der einzelnen Elemente tritt eine auf Komprimierung der Takte 11-21 gerichtete Verklammerung. Der B-Dur-Septakkord in Takt 112 wird mit einem Es-Dur-Akkord als Dominante nach As verschränkt, das wiederum in Takt 114 erscheint. Der Ges-Dur-Septakkord vor dem Überleitungsthema wird hier nicht wie in der Exposition zum übermäßigen Quintsextakkord umgedeutet, um anschließend in das B-Dur des Überleitungskanons überzuleiten, sondern ganzschlüssig nach Ces-Dur aufgelöst. Indem Brahms das tiefchromatische CesDur in Takt 124/125 wieder nach Es-Dur hebt, vermeidet er eine exakte Wiederholung des Überleitungsthemas. In Takt 138 folgen unverändert, nunmehr auf der I. statt auf der V. Stufe, der Seitensatz sowie die Schlussgruppe.

\section{Coda als Resümee}

Infolge der Idee, Reprise und Coda als modifizierte Wiederholung von Exposition und Durchführung darzustellen, ergeben sich bei der Bestimmung des Beginns der Coda die gleichen Schwierigkeiten wie im Falle des Durchführungsbeginns. Oberflächlich betrachtet liegt ein Beginn der Coda in Takt 154 nahe. Aber auch hier wird, analog zur Durchführung, der Beginn durch das zweifache Auftreten des Oktavregelmodells verschleiert. Für einen Beginn der Coda in Takt 150 spricht außerdem, dass die Takte 154ff. - ganz anders als am Durchführungsbeginn - eine variierte Wiederholung der Takte $11 \mathrm{ff}$. darstellen und damit nicht auf den Anfang des Satzes verweisen. Brahms integriert hier

22 Vgl. dazu Webster 1990, 64.

23 Vgl. Webster 1990, 50f. und Reiter 2000, 266.

24 Ein Beispiel hierfür ist die Reprise in op. 115, in der der gesamte erste thematische Komplex am Reprisenbeginn eliminiert wird. Takt 136 beginnt analog zur Exposition: Zwei Takte später in Takt 138 setzen die Geigen analog zu Takt 14 ein und süberspringen` damit zwölf Takte. Ein weiteres Beispiel findet sich in op. 111 in Takt $106 \mathrm{ff}$. 
in die Basslinie noch einen steigenden Fauxbourdonsatz (ab T. 156), der an das harmonische Gerüst des Seitensatzes erinnert. Damit deutet sich bereits die Idee an, die kompositorischen Problemstellungen des Satzes in der Coda zusammenzufassen.

Das wesentliche Argument, die Coda als komprimierte Zusammenfassung des Satzverlaufs aufzufassen, ist jedoch, dass sich die beiden oben erläuterten übergreifenden Materialebenen des Satzes, Oktavregelmodell und charakteristische Wendungen (vgl. Beispiel 1 und 2), am verschleierten Beginn der Coda erstmals überlappen (T. 149ff.) anstatt wie bisher im Satzverlauf unabhängig voneinander verlaufen. Ein weiterer zusammenfassender Aspekt sind die von Brahms quasi zitathaft aufgerufenen Quintfallsequenzen. Während Sequenzen im Satzverlauf ansonsten vielfach durch variative Prozesse verschleiert erscheinen, wird die finale diatonische Quintfallsequenz (T. 162ff.) quasi snacktı ausgestellt. In ihrer satztechnischen Simplizität stellt sie - durch die Generalpause und das langsamere Tempo noch verstärkt - eine Ausnahme in der komplexen und durchgearbeiteten Struktur des Satzes dar. ${ }^{25}$

Auf die srichtige` Auflösung des übermäßigen Quintsextakkords in Takt 161 in den Quartsextakkord über $b$ folgt zunächst keine Auflösung nach Es-Dur. Letztere wird erst am Ende der Quintfallsequenz erreicht, so dass hier die Ebene der charakteristischen harmonischen Wendungen mit der insbesondere die Durchführung dominierenden Sequenztechnik kombiniert wird. Ab Takt 158 kombiniert die vorgelagerte Quintfallsequenz beide Abschnitte des Überleitungsthemas (Kanon und homophone Fortspinnung) und überführt damit auch die musikalische Substanz des Überleitungsthemas in einen sequenziellen Kontext. Die Klarinettenmelodie in Takt 158f. ist eine sequenzielle Fortspinnung des Überleitungskanons aus Takt 30 bzw. Takt 128. Die durchlaufende Achtelbewegung die im Klavier zwischen Diskant und Bass alterniert hat eine Betonung jedes Viertels im Gegensatz zur Betonung der schwachen Zählzeiten in Takt 30 bzw. 128 zur Folge. Sie erweist sich als Variation der Begleitfigur des Überleitungskanons, und mündet in Takt 160/161 in einen Dominantseptakkord auf H. Dieses harmonische Stagnieren hat seine Entsprechung in dem unentschlossenen Verebben der Klarinettenmelodie, was hier auf vergleichbare Gesten am Ende des Hauptsatzes (T. 21 und T. 119) und die Verschleierungen von Durchführungs- und Codabeginn (T. 55 und T. 153) erinnert. Nach der Es-Kadenz in Takt 166 folgen - wie im verwandten Zentralteil der Durchführung (T. 78ff.) - zwei plagale Wendungen in Takt 168/169 sowie eine doppelte Es-Dur-Kadenz in Takt 170/171. Die letzten drei Takte sind ein bloßes >Auskomponieren<, eine sApotheose der Es-Dur-Tonika als reine Klangerscheinung. Auf diese Weise ruft Brahms in der Coda, wenn auch in stark modifizierter Weise, alle wesentlichen Elemente des Satzes in Erinnerung.

$25 \mathrm{Vgl}$. auch die detaillierte Analyse der beiden Sequenzen und deren Interpretation im Hinblick auf den gesamten Satzverlauf bei McClelland. Die Sequenzen werden dort unter der Überschrift »Transcendent and Culminating Sequences« rubriziert (2012, 176ff.). 
Fazit

Insgesamt ist der Satz durch die Zurücknahme dramatischer Entwicklungsmomente geprägt. Der Reprisenbeginn büßt dadurch vordergründig an Bedeutung ein, bleibt jedoch durch das komplexe Reprisenvorfeld einer der spannungsreichsten Momente des Satzes. Dieser Situation vergleichbar steht auch in der Coda die beschriebene Zunahme an Komplexität einer dynamischen Zurücknahme gegenüber. Mit der zusammenfassenden Funktion der Coda bleibt Brahms zwar beim Konzept eines finalorientierten Satzverlaufs, der am Ende eines kompositorischen Prozesses ıLösungen` von kompositorischen Problemstellungen präsentiert. In klanglich-dynamischer Hinsicht jedoch wird diese Lösung in einer Geste der Zurücknahme präsentiert.

Brahms unterwirft die Sonatenform graduellen Veränderungen und deutet einige konstitutive Elemente dieser Form durch konzentrierteres Auftreten nur noch an. Dabei begreift er den Sonatensatz nicht als beliebig ausfüllbares formales Dispositionsschema, sondern als ein Formprinzip, das die Erzeugung weiträumiger musikalischer Zusammenhänge ermöglicht, die er durch Rückschluss-Situationen und subkutane Wiederholungsstrukturen artikuliert. Im Hinblick auf Sequenzbildungen zeigt sich neben deren Unregelmäßigkeit das Prinzip, Sequenzflächen zu verkürzen und zu verdichten, so dass regelmäßige und längere Sequenzbildungen - wie beispielsweise in der Coda des ersten Satzes - als quasi-zitathafte Erinnerungen an frühere Konventionen auffallen. Diese Erinnerungen wirken in der Spätzeit der Dur-Moll-Tonalität wie ein melancholischer Abgesang.

\section{Literaturverzeichnis}

Adrian, Jack (1990), »The Ternary-Sonata Form«, Journal of Music Theory 34, 57-80.

Bach, Carl Philipp Emanuel (1753/1762), Versuch über die wahre Art das Clavier zu spielen, Berlin, Reprint beider Teile, hg. von Wolfang Horn, Kassel: Bärenreiter 2003.

Boestfleisch, Rainer (2001), „Überlegungen zum ersten Satz der Klarinettensonate op. 120/1 von Johannes Brahms", in: Die Kammermusik von Johannes Brahms. Tradition und Innovation. Bericht über die Tagung Wien 1997, hg. v. Gernot Gruber, Laaber: Laaber, 285-306.

Cone, Edward T. (1968), Musical Form and Musical Performance, New York: Norton.

Dahlhaus, Carl (1974), „Zur Problemgeschichte des Komponierens", in: Ders., Zwischen Romantik und Moderne: Vier Studien zur Musik des späteren 19. Jahrhunderts (= Berliner musikwissenschaftliche Arbeiten 7), München: Katzbichler, 40-71.

Edelmann, Bernd (2013), »Zwei Sonaten für Klarinette (oder Viola) und Klavier f-Moll und Es-Dur", in: Johannes Brahms. Interpretationen seiner Werke, Bd. 2, hg. von Claus Bockmaier und Siegfried Mauser, Laaber: Laaber, 878-892.

Falke, Gustav-Hans (1997), Johannes Brahms. Wiegenlieder meiner Schmerzen - Philosophie des musikalischen Realismus, Berlin: Lukas-Verlag. 
Krämer, Ulrich (2009), „Kammermusik mit Bläsern«, in: Brahms-Handbuch, hg. von Wolfgang Sandberger, Kassel und Stuttgart: Bärenreiter und Metzler, 457-473.

McClelland, Ryan (2012), „Sequence as Expressive Culmination in the Chamber Music of Brahms", in: Expressive Intersections in Brahms. Essays in Analysis and Meaning, hg. von Heather Platt und Peter H. Smith, Bloomington u. a.: Indiana University Press, 147-185.

Reiter, Elisabeth (2000), Der Sonatensatz in der späten Kammermusik von Brahms: Einheit und Zusammenhang in variativen Verfahren, Tutzing: Schneider.

Schönberg, Arnold (1976a), „Brahms der Fortschrittliche«, in: Stil und Gedanke: Aufsätze zur Musik (= Gesammelte Schriften 1), hg. von Ivan Vojtech, Frankfurt a.M.: Fischer, 35-71.

(1976b), „Kriterien für die Bewertung von Musik», in: Stil und Gedanke: Aufsätze zur Musik (= Gesammelte Schriften 1), hg. von Ivan Vojtech, Frankfurt a. M.: Fischer, 123-133.

Schmidt, Christian-Martin (1971), Motivisch-thematische Vermittlung in der Musik von Johannes Brahms dargestellt an der Klarinettensonate f-moll, Op. 120/1, München: Katzbichler.

(1983), Johannes Brahms und seine Zeit (Große Komponisten und ihre Zeit), Laaber: Laaber.

_ (1994), Johannes Brahms (= Reclam Musikführer), Stuttgart: Reclam.

_ (2000), Johannes Brahms. Versuch über die musikalische Selbstreflexion, Wilhelmshaven: Noetzel.

Sprick, Jan Philipp (2012), Die Sequenz in der deutschen Musiktheorie um 1900, Hildesheim: Olms.

Stahmer, Klaus Hinrich (1983), „Der eigenwillige Traditionalist - Das kammermusikalische Spätwerk von Johannes Brahms", in: Johannes Brahms. Leben und Werk, hg. von Christine Jacobsen, Wiesbaden: Breitkopf \& Härtel.

Webster, James (1990), „The general and the particular in Brahms's later sonata forms", in: Brahms studies. Analytical and historical perspectives, hg. von Georges Bozarth, Oxford: Oxford University Press, 49-78. 